\title{
Age-Associated Impairments in a Test of Attention: Evidence for Involvement of Cholinergic Systems
}

\author{
D. N. C. Jones, ${ }^{1, a}$ J. C. Barnes, ${ }^{2}$ D. L. Kirkby, ${ }^{1}$ and G. A. Higgins ${ }^{1,2}$ \\ 'Glaxo Unit for Behavioral Psychopharmacology, Division of Biosciences, University of Hertfordshire, Hatfield, Herts, \\ AL10 9AB and 'Pharmacology 2, Glaxo Research and Development Ltd., Ware, Herts, SG12 ODP, United Kingdom
}

We trained three groups of rats, young ( $\mathrm{Y} ; 3$ months at the start of study), middle aged (MA; 15 months), and aged (AG; 22 months), in the serial five-choice serial reaction time task, a test of attention. There were clear age-related differences in task acquisition: $Y$ acquired the task quicker than MA rats, which learned faster than $A G$ rats. $A$ subgroup of $A G$ rats $[A G(I)]$ could not reach criterion $(>80 \%$ correct, $<20 \%$ omissions under standard conditions of 0.5 sec stimulus duration, 5 sec limited hold). Accordingly, they were tested under conditions of 1 sec stimulus duration. Having acquired the task, under standard conditions both MA and AG groups were slower to make a correct response but not to collect the food reward. Furthermore, parameter changes, particularly reductions in stimulus duration and intensity, revealed further age-related changes in accuracy. Following completion of these studies, animals were trained in a simpler one-choice task. Importantly, reducing stimulus duration/intensity in this task revealed no differences between $Y$ and MAAG groups, although $A G(I)$ rats were impaired. This dissociation between MAAG impairments in the one- and five-choice task suggests that these animals may show attentional deficits compared with $Y$ rats, which are independant of changes in sensory (visual), motor function, or motivation. Finally, the MA deficit in attention was partially reversed by tacrine pretreatment (3 $\mathrm{mg} / \mathrm{kg})$. Also scopolamine (0.01-0.075 $\mathrm{mg} /$ $\mathrm{kg})$ and mecamylamine $(0.3-5 \mathrm{mg} / \mathrm{kg})$ pretreatment impaired choice accuracy of MA but not $Y$ rats. Taken together, the drug studies imply that the attentional deficits may at least be partially due to changes in cholinergic function.

[Key words: age, rat, serial five-choice reaction time task, tacrine, selective attention, scopolamine, mecamylamine]

Impaired attentional processes results in severe cognitive and behavioral dysfunctions (see Sarter, 1994). Deficits in attention are associated with age-related decrements in human memory (Craik and Simon, 1980; Craik and Byrd, 1982) and comprise some of the cognitive deficits associated with Alzheimer's disease (AD; Capitani et al., 1988; Freed et al., 1989; Grady et al., 1989; Parasuraman et al., 1992; Parasuraman and Martin, 1994). Indeed, impairments in various parameters of attention may rep-

Received Apr. 19, 1995; revised June 20, 1995; accepted July 5, 1995.

Correspondence should he addressed to Dr. Guy Higgins, Glaxo Wellcome Medicine Research Centre, Gunnels Wood Road, Stevenage, Herts, SGI 2NY, UK.

Present address: Psychiatry Research, SmithKline Beecham plc, New Frontiers Science Park, Harlow, Essex, UK

Copyright $\bigcirc 1995$ Society for Neuroscience $0270-6474 / 95 / 157282-11 \$ 05.00 / 0$ resent the first signs of neocortical dysfunction in AD (Parasuraman and Haxby, 1993). Many of the neural systems most affected in $\mathrm{AD}$, including the basal forebrain cholinergic system, play significant roles in attentional processes (see Parasuraman et al., 1992).

However, there have been few attempts to develop tests of attention and model the attentional deficits associated with aging and $\mathrm{AD}$ in animals. One such test is the five-choice serial reaction time (5-CSRT) task, where rats have to detect brief flashes of light presented randomly in one of five spatial locations (Carli et al., 1983). Robbins and co-workers have examined attentional effects following disruption of specific neurotransmitter systems in this task (Carli et al., 1983; Cole et al., 1989; Robbins et al., 1989; Muir et al., 1992a,b; 1994). The cholinergic system is particularly important for maintaining high levels of accuracy in this task. For example, disruption of the basal forebrain cholinergic system, degeneration of which is a feature of normal aging (McGeer et al., 1984; Flood and Coleman, 1988; Vogels et al., 1990) and of the neuropathology of AD (Perry et al., 1978; Whitehouse et al., 1982; Coyle et al., 1983), impaired accuracy in this task. This impairment was attenuated by treatment with physostigmine or cholinergic-rich cortical grafts (Robbins et al., 1989; Muir et al., 1992a, 1994).

There are many reports of age-related differences in cognitive performance in animals (Bartus et al., 1980; Albert, 1988; Gallagher and Pellymounter, 1988; Barnes, 1990; Gower and Lamberty, 1993; McGaughy and Sarter, 1995) Given that aged rats show some degeneration of the basal forebrain cholinergic system (Fischer et al., 1991; Flood and Coleman, 1988; Smith et al., 1993), it is expected that they would be impaired in tests of attention. Moore et al. (1992) investigated the effects of aging upon performance of 4, 12, and 18-month-old Fischer-344 rats in a test of vigilance. Performance was assessed in simple- and choice-reaction time tasks (SRTT and CRTT, respectively) and appeared to successfully detect an age-related decrease in vigilance. However, the CRTT, which required rats to locate the signal and decide among two response alternatives, failed to separate age-related differences to a greater extent than the SRTT, which did not require a decision about the correct response to make.

The aim of the present study was to determine the effects of aging upon performance of rats in the 5-CSRT task. Three groups of rats $(3,15$, and 22 months old at the start of the experiment) were trained initially in the 5-CSRT task, and the effects of age upon the acquisition, baseline performance, and sensitivity to increases in the attentional load were determined. Subsequently, the performance of the rats in a simplified version 
of the task, the simple reaction time task (SRTT), in which the light stimulus appeared in a central, predictable location was analyzed. Finally, the effects of the cholinesterase inhibitor, tacrine, and the cholinergic antagonists scopolamine and mecamylamine were determined. Tacrine was selected as it has been reported to attenuate some of the performance deficits in a computeriized, touch-screen version of the 5-CSRT task in AD patients (Sahakian et al., 1993).

\section{Materials and Methods}

Animals

The subjects were 13 young (Y), 14 middle-aged (MA), and 18 aged (AG) male, hooded Lister rats $(3,15$, and 22 months old, respectively, at the start of the experiment). Only rats that appeared to be healthy and showed no overt signs of ill health (e.g., tumors, cataracts, or locomotor disabilities) were trained. Accordingly, two additional aged rats were excluded at the start of the experiment. Very occasionally, oral antibiotic treaments (Terramycin. Glaxo. UK) were given during these studies for the treatment of mild chest infections. Treated rats were withdrawn from studies and reintroduced only upon reaching good health. Pairs of age matched rats were housed in a room controlled for constant temperature $\left(22 \pm 1^{\circ} \mathrm{C}\right)$ and humidity $(50 \%)$. Water was available ad libitum, but food was restricted to that earned during the test [maximum of $96 \times 45 \mathrm{mg}$ Formula $P$ purified pellets (Noyes, Lancaster, $\mathrm{NH})]$ and 45 min access to standard rodent chow (SDS, Witham, Essex, UK) at the end of the test day (approximately 1630-1730 h). All testing was conducted during the light phase of the light/dark cycle (lights on: 0700-1900 h).

\section{Apparatus}

The apparatus (Paul Fray Ltd., Cambridge, UK) used was very similar to that described by other workers (Carli et al., 1983). It consisted of eight $25 \times 25 \mathrm{~cm}$ aluminium chambers, each with a curved rear wall. Set into the curved wall were nine, $2.5 \mathrm{~cm}$ square holes, $4 \mathrm{~cm}$ deep and $2.5 \mathrm{~cm}$ above a wire mesh floor. Each hole had an infrared photocell beam monitoring its entrance and was illuminated by a $3 \mathrm{~W}$ bulb located at the rear of the hole. For the five-choice task, holes $2,4,6$, and 8 (from the left hand side) were blocked with metal covers; in the simple reaction time task, all the holes except number 5 were blocked. A food pellet dispenser delivered pellets into a magazine at the front of the chamber. Access was gained to the magazine through a perspex panel, the operation of which was monitored by a micro switch. The chamber was illuminated by a $3 \mathrm{~W}$ house light mounted in the roof alongside a small general purpose loud-speaker through which bursts of white noise could be delivered (white noise generator 530, Camden Instruments Ltd., Loughborough, UK). The animal was introduced to the chamber through a perspex door in the top half of the front wall. The chamber was contained within a sound-attenuating wooden box. Ventilation was by means of a fan providing a low level of background noise which masked extraneous sounds. On-line control of the apparatus and data collection was performed using a microcomputer [Archimedes 440/1 programmed using Arachnid (Paul Fray Ltd., Cambridge, UK)].

\section{Behavioral training}

Magazine training. The training methods adopted were a modified version of those used by other workers (e.g.. Carli et al., 1983). Rats were introduced to the restricted diet 1 week prior to training with standard rodent chow supplemented with the food reward pellets. On the first 2 $\mathrm{d}$ of training, rats were placed in the chambers for $15 \mathrm{~min}$ with the houselight on and allowed free access to approximately 30-40 food pellets placed in the food tray. On the next $2 \mathrm{~d}$, rats were placed in the chamber for $15 \mathrm{~min}$ and a food pellet was delivered every $15-30 \mathrm{sec}$ into the magazine.

Five-choice serial reaction time task. Initially, all animals were trained in the five-choice task. Training began on the fifth day. The start of each training session was signalled by illumination of the houselight and delivery of a "free" food pellet to the magazine. Opening the magazine panel to retrieve the pellet initiated the first trial. After a fixed delay (intertrial interval or ITI), the light at the back of one of the open holes was illuminated [575 lux, measured with a hand-held digital light meter (testoterm 0500, RS Components, Corby, UK)] for a predetermined duration (stimulus duration or SD). Responses (nose pokes) into the hole when illuminated (correct response) and for a short period after (limited hold or $\mathrm{LH}$ ) were rewarded with the delivery of a food pellet. Additional responses in this hole (perseverative responses) resulted in a $5 \mathrm{sec}$ period of darkness (time out). A response in any other hole (incorrect response), failure to respond within the LH period (omission), or responses in the holes during the ITI (premature responses) were punished with a $5 \mathrm{sec}$ time out. Responses during the time-out period caused the time out to be restarted. Opening of the magazine panel to retrieve a food pellet or after a time-out period initiated the next trial. The light stimulus was presented randomly in each of the five holes on an equal number of occasions. Each daily session terminated after 100 trials or $30 \mathrm{~min}$ of testing, whichever was sooner. At the end of the session, all lights were extinguished and further responses were ineffective.

Task acquisition. In order to allow comparisons between the different age groups on the acquisition of this task, the training schedule was divided into several stages. At all of these stages, rats were required to initiate 100 trials within the $30 \mathrm{~min}$ period and miss less than $20 \%$ of these before passing on to the next training stage. The target parameters were: SD, $0.5 \mathrm{sec}$; LH, $5 \mathrm{sec}$; ITI, $5 \mathrm{sec}$. For the first two sessions, SD and $\mathrm{LH}$ were $60 \mathrm{sec}$ and ITI and time out were $3 \mathrm{sec}$ (increased to 5 sec on the third session). Once the rats were getting more than $90 \%$ of trials correct on three consecutive occasions, the SD and LH were decreased to $30 \mathrm{sec}$. After two consecutive sessions at the above criterion. the SD and LH were reduced to $10 \mathrm{sec}$ for one session. This did not impair performance in any rats; therefore, SD and LH were reduced to $5 \mathrm{sec}$ until two consecutive sessions at above $80 \%$ accuracy were completed. SD was reduced to $1 \mathrm{sec}$ until two consecutive sessions at above $80 \%$ accuracy, when it was reduced to $0.5 \mathrm{sec}$. Rats were considered trained when accuracy was greater than $80 \%$ at the target conditions on three consecutive sessions. However, after 45 training sessions, a proportion of the aged rats $(n=11)$ still had not reached criterion at 0.5 sec SD. Therefore, these rats were assigned to a separate group with all subsequent testing using a SD of $1 \sec [A G(1 \mathrm{sec})]$.

Behavioral challenges. A series of manipulations of the stimulus parameters was employed posttraining in a manner similar to that used by Robbins et al. (1989). In all cases, rats were presented with 100 trials ( 25 of each trial type presented in a pseudorandom order) and allowed $40 \mathrm{~min}$ to complete the session. The exception was variable, longer ITI duration, which was 80 trials, with 20 of each type. Behavioral challenges occurred on Tuesdays and Fridays over a period of 3-4 weeks. Each test day was preceded by at least two training sessions under standard conditions (SC). The challenges were made in the following order: (i) variable, stimulus duration (SD, $0.05,0.15,0.25$, or $0.5 \mathrm{sec}$ for all groups except for $\mathrm{AG}(1 \mathrm{sec})$, which was $0.15,0.25,0.5$, or I $\mathrm{sec}$ ); (ii) variable, stimulus intensity (SI, $575,82,45$, or 16 lux, i.e. software values of $10,3,2$, or 1 ); (iii) variable, ITI duration (short: 2 , 3,4 , or $5 \mathrm{sec}$; and long: $5,6,7$, and $8 \mathrm{sec}$ ); (iv) interpolation of a white noise distractor $[105 \mathrm{~dB}$ (measured with a digital sound level meter type D-1422C, RS Components, Corby, UK), $0.5 \mathrm{sec}$ duration] at varinus time points into the normal $5 \mathrm{sec}$ ITI (no noise, $0,2.5$, or $5 \mathrm{sec}$ ): (v) combinations of variable SD [0.15 or $0.5 \mathrm{sec}-0.25$ and $1 \mathrm{sec}$ for $\mathrm{AG}(1 \mathrm{sec})]$ - with variable SI ( 45 or 575 lux).

Performance measures and data analysis. The performance measures were \%correct (i.e., the percentage of completed trials that were correct), \%omissions (i.e., the percentage of trials which were missed) latency to make a correct response (correct latency), latency to retrieve the food reward (magazine latency), number of premature and perseverative responses, and the number of magazine panel pushes. For the sake of brevity, only data for \%correct is presented under each stimulus condition as well as collapsed over the total trials. All other performance measures are presented for total trials only. Data was analyzed by ANOVA following transformation of data, where appropriate (\%correct/omissions: arcsin; latencies: log; premature and perseverative responses and panel pushes: square root). Post hoc analysis was carried out using Tukey's $t$ test.

Simple reaction time task. Upon completion of the above series of experiments rats were trained in the simple reaction time task (SRTT) in which the light stimulus only appeared in the central location each time (hole 5). All other holes were blocked. In the first session, the SD was $60 \mathrm{sec}$ and was gradually reduced over five sessions to $0.5 \mathrm{sec}$, except the $\mathrm{AG}(1 \mathrm{sec})$ group, which were reduced to a final SD of $1 \mathrm{sec}$. To avoid complete temporal predictability of the light stimulus that would raise the possibility that rats could respond with a high level of accuracy without having to detect the stimulus, the ITI was varied over 
Figure 1. Effect of age on acquisition of the serial five-choice task. ( $\square$ Y group, (crosshatched) MA group, (slanted rules) $\mathrm{AG}(0.5 \mathrm{sec}),(\square) \mathrm{AG}(1$ $\mathrm{sec}) . A$. The number of sessions to reach a criterion of $80 \%$ correct with < $20 \%$ omissions with the stimulus duration of $60 \mathrm{sec}, 1 \mathrm{sec}, 0.5 \mathrm{sec}$. $B$, The total number of training sessions required by each age group from food shaping to the final critereon of $>80 \%$ correct $<20 \%$ omissions at $0.5 \mathrm{sec}$ stimulus duration (see Materials and Methods for further detail). ${ }^{*} p<0.05$ ${ }^{* *} p<0.01$ vs Y group (Tukeys $t$ test).

(1)
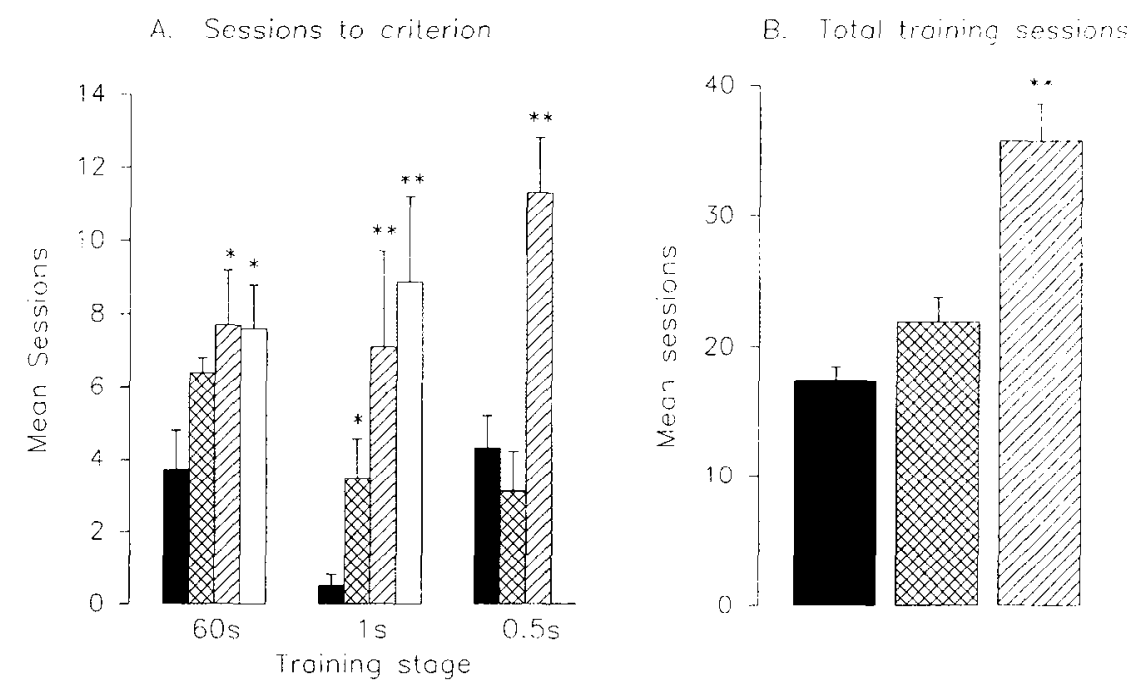

a narrow range $(4.25,4.75,5.25$, or $5.75 \mathrm{sec})$ and the LH was reduced to $2 \mathrm{sec}$. Rats were tested under these conditions for five further sessions within which time performance had stabilized.

Behavioral challenges. From the series of behavioral challenges carried out in the five-choice task, it was apparent that reducing the SD and SI impaired accuracy in an age-dependent manner. Therefore, the effects of reducing SD and SI were tested in the SRTT. However, because of the requirement for balanced combinations of stimulus characteristics and variable ITI duration, the software limited changes to only two SD or SI levels combined with two ITI durations (in this case 4.5 and $5.5 \mathrm{sec})$ : (i) variable, SD [0.15 or $0.5 \mathrm{sec}$, except $\mathrm{AG}(1 \mathrm{sec})$ : $0.25 .1 \mathrm{sec}$; (ii) variable, SI ( 45 or $575 \mathrm{lux}$ ).

Performance measures. The performance measures were total correct responses, \%omissions, latency to make a response (response latency), magazine latency, number of premature and perseverative responses, and the number of magazine panel pushes. Under SC, the data was collapsed across all four ITI durations. However, when challenged with reduced SD or SI, the data for total correct responses for each SD or SI condition was collapsed across hoth the ITI durations. For all other measures, the data was collapsed across all conditions.

Influence of tacrine $13 \mathrm{mg} / \mathrm{kg}$ s.c.) upon performance in the fivechoice serial reaction time task. After testing in the SRTT, the rats from $\mathrm{MA}$ and $\mathrm{Y}$ only were retrained in the 5-CSRT task over a 2 week period until a stable level of performance was reached. Using a Latin square design in the MA rats, tacrine ( 3 ming/kg s.c.) or vehicle (VEH, $0.9 \%$ $\mathrm{w} / \mathrm{v}$ saline) were administered at $1700 \mathrm{~h}$ the night before, and $45 \mathrm{~min}$ prior to, testing under conditions of combinations of variable SD $(0.15$ or $0.5 \mathrm{sec}$ ) with variable SI (45 or $575 \mathrm{lux}$ ). Rats were tested on Wednesday with training under SC on all other weekdays. Data was analyzed as a two-factor repeated-measures ANOVA (drug, trial type). Post hoc analysis was carried out using Tukey's $t$ test. Previous studies (Jones et al., 1993) showed that performance deficits under the comhination condition remained stable under this protocol.

The influence of scopolamine $(0.01-0.075 \mathrm{mg} / \mathrm{kg} \mathrm{s.c.)}$ and mecamylamine $(0.3-5 \mathrm{mg} / \mathrm{kg}$ s.c.). Approximately 10 months after the beginning of training the effects of scopolamine $(0.01-0.075 \mathrm{mg} / \mathrm{kg} \mathrm{s.c.})$ and then mecamylamine $(0.3-5 \mathrm{mg} / \mathrm{kg}$ s.c.) were assessed in groups $\mathrm{Y}$ and MA under SC. Drugs were administered 30 min prior to testing on Tuesdays and Thursdays with training under SC on other days. Rats received all doses of each drug with a 2 week period between the two studies. Data was analyzed using a two-factor ANOVA (age and drug). Post hoc analysis was carried out using Tukey's $t$ test.

Locomotor activity response to a novel environment. Immediately after completing the SRTT studies, the locomotor activity (LMA) of seven to eight randomly selected rats from each age group was determined as part of the continuing investigation of the role of sensorimotor deficits in task performance. Eight polypropylene activity chambers (31 $\times 19 \times 18 \mathrm{~cm} ; \mathrm{L} \times \mathrm{W} \times \mathbf{H})$ were used. Activity was measured by the animal interrupting an array of photoelectric beams positioned $3 \mathrm{~cm}$ and $11 \mathrm{~cm}$ above the cage floor. Following a $60 \mathrm{~min}$ habituation period to the laboratory, rats were placed individually into the boxes and activity was measured at $5 \mathrm{~min}$ intervals for a $60 \mathrm{~min}$ period. The animals had no previous experience of this apparatus. Data for total activity counts for each 5 min time bin were analyzed using a two-way ANOVA (factors: time bin and age), and a one-way ANOVA was carried out on data for total activity for the $60 \mathrm{~min}$ period.

\section{Results}

The rat weights at the start of training were: $567 \pm 17 \mathrm{gm}$ [AG $(0.5 \mathrm{sec})], 538 \pm 11 \mathrm{gm}[\mathrm{AG}(1 \mathrm{sec})], 536 \pm 18 \mathrm{gm}(\mathrm{MA})$, and $369 \pm 7 \mathrm{gm}(\mathrm{Y})$. At the end of the training period (approximately 12 weeks later), the weights were: $456 \pm 17 \mathrm{gm}$ $[\mathrm{AG}(0.5 \mathrm{sec})], 426 \pm 10 \mathrm{gm}[\mathrm{AG}(1 \mathrm{sec})], 426 \pm 9 \mathrm{gm}(\mathrm{MA})$, and $371 \pm 6 \mathrm{~g}(\mathrm{Y})$. Throughout the course of these studies, certain aged rats were withdrawn because their health deteriorated to levels that might affect task performance. Therefore, at each stage of this work the group $n$ is presented to reflect this adjustment.

\section{Five-choice serial reaction time task}

\section{Task acquisition}

The data for the rate of acquisition of the five-choice task is illustrated in Figure 1. Overall, there was a significant main effect of age upon the mean total sessions to complete training to SC. [excluding group AG(1 sec), $F(2,29) 21.1, p<0.0001$, Fig. $1 b$ ]. Virtually all of the rats reached criterion at the 30,10 , and $5 \mathrm{sec}$ stages immediately (data not shown). There was a main effect of age $[F(2,87) 23.7, p<0.001]$ upon number of sessions to criterion at each training stages (i.e., 60,1 , and $0.5 \mathrm{sec}$, Fig. $1 a)$ with $\mathrm{AG}(0.5 \mathrm{sec})$ significantly worse than $\mathrm{Y}$ at each stage $(p<0.05-0.01)$ and MA worse than $\mathrm{Y}$ at the $1 \mathrm{sec}$ stage $(p<$ 0.05 ). A direct comparison between all four groups could only be made at the 60 and $1 \mathrm{sec}$ stages: $\mathrm{AG}(1 \mathrm{sec})$ were significantly worse than $\mathrm{Y}$ at both these stages $(p<0.05-0.01)$, but were not different from $\mathrm{AG}(0.5 \mathrm{sec})$.

\section{Performance under standard conditions (SC)}

The performance of rats under SC is shown in Figure $2 a-d$. Rats were tested once daily for the 5 d following division of the aged rats into two groups. There were main effects of age upon \%correct $[F(3,204) 3.6, p<0.05$, Fig. $2 a]$, \%omissions $[F(3,204)$ $13.7, p<0.0001$, Fig. $2 b]$, correct latency $[F(3,204) 30.3, p<$ 0.0001 , Fig. $2 c]$, magazine latency $[F(3,204) 5.8, p<0.01$, Fig. $2 d]$, premature responses $[F(3,204) 5.6, p<0.01]$, and panel pushes $[F(3,204) 3.7, p<0.05]$, but not perseverative responses. 

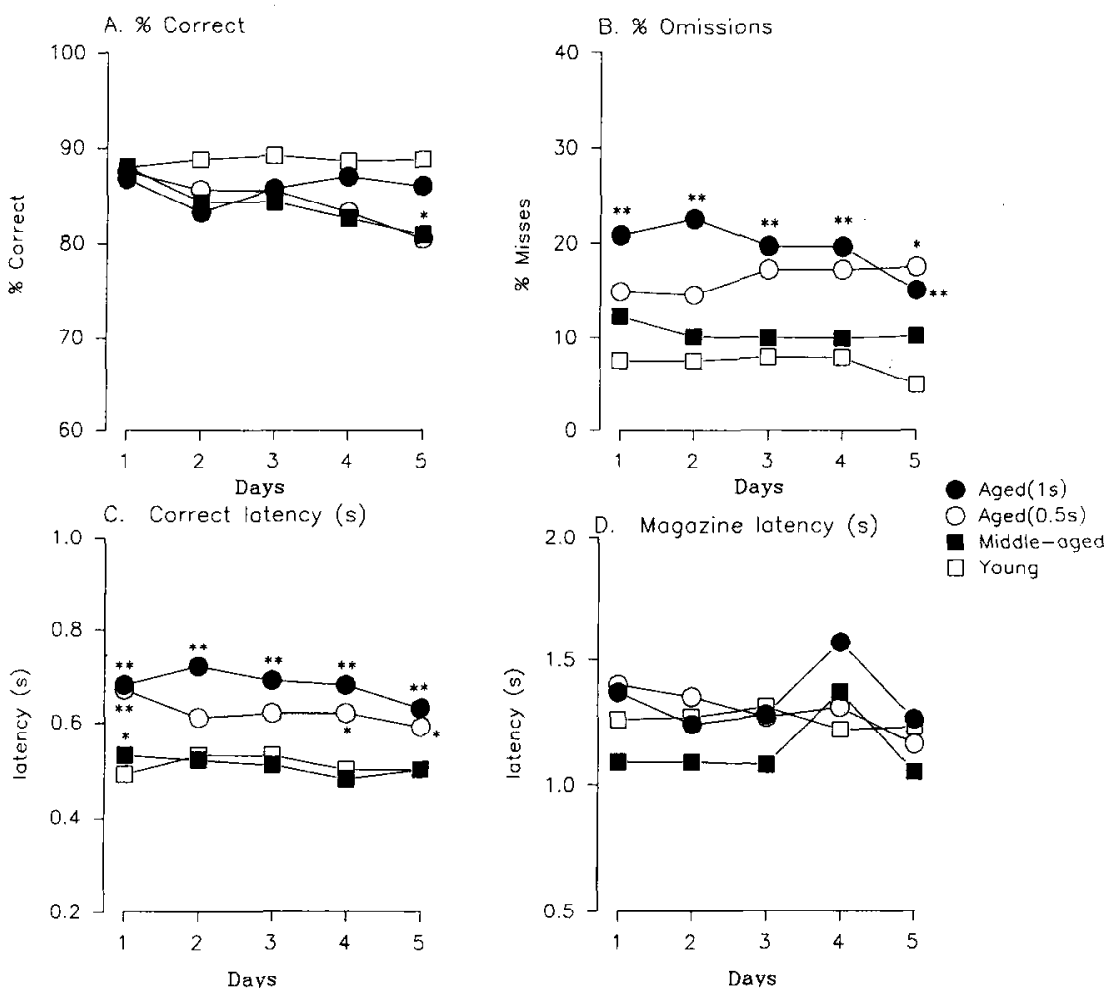

Figure 2. Effect of age on performance over $5 \mathrm{~d}$ continual testing under standard test conditions in the serial five-choice task. ( $\square$ ) Y group, ( $\square$ ) MA group, (O) $\mathrm{AG}(0.5 \mathrm{sec})$, (O) $\mathrm{AG}(1$ sec). Data shown is for $(A)$ percentage correct, $(B)$ percentage omissions, $(C)$ correct latency (sec), $(D)$ magazine latency $(\mathrm{sec}) .{ }^{*} p<0.05 * * p<0.01$ vs Y group (Tukeys $t$ test).

The reduced \%correct of the $\mathrm{AG}(0.5)$ and MA group compared to $\mathrm{Y}$ rats on day 5 (Fig. $2 a$ ) was unusual and not reflective of the general performance of these groups under $\mathrm{SC}$, which was similar to Y rats during the course of these studies. Rather, the clearest effects of age were upon \%omissions and correct latency. For magazine latency, the age-related difference appeared to be the result of a trend towards MA rats retrieving the food reward more quickly than other groups.

\section{Behavioral challenges}

Variable, reduced stimulus duration. This experiment used 7 rats from $A G(0.5 \mathrm{sec}), 11$ rats from $A G(1 \mathrm{sec}), 14$ rats from MA, and 13 rats from $Y$. The effects of reducing SD on accuracy are illustrated in Figure $3 a$. Because of the differences in SDs used for $\mathrm{AG}(1 \mathrm{sec})$, only data for $\mathrm{AG}(0.5 \mathrm{sec}), \mathrm{MA}$, and $\mathrm{Y}$ were included in the analysis of \%correct data by SD. There were main effects of age $[F(2,124) 6.4, p<0.01]$ and SD $[F(3,124)$ $37, p<0.0001]$ upon \%correct, reflecting that MA and $\mathrm{AG}(0.5$ sec) performed significantly worse than $Y$ rats at particular SD, notably $0.15 \mathrm{sec}$.

Further analysis examined data collapsed across the four different SDs and included group $\mathrm{AG}(1 \mathrm{sec})$. There was no effect of age upon overall \%correct $[F(3,41) 2.3, p=0.09]$, although there was a trend for $\mathrm{AG}(0.5 \mathrm{sec})$ and MA to show lower choice accuracy compared to $\mathrm{Y}$ rats (Table 1). There was an age-related increase in overall \%omissions $[F(3,41)=5, p<0.001]$, such that $\mathrm{AG}(1 \mathrm{sec})$ rats missed significantly more trials than both MA and Y rats $(p<0.01$; Table 1$)$. There was a near-significant trend towards an age-related increase in correct latency $[F(3,41)$ $=2.7, p=0.057]$, with $\mathrm{AG}(0.5 \mathrm{sec})$ and $\mathrm{AG}(1 \mathrm{sec})$ slower than $\mathrm{Y}$ rats (see Table 1).

Variable, reduced stimulus intensity. This experiment used 7 rats from $A G(0.5 \mathrm{sec}), 11$ rats from $A G(1 \mathrm{sec}), 14$ rats from MA, and 13 rats from $\mathrm{Y}$, and results are shown in Figure $3 b$. There were significant main effects of age $[F(3,164)=8.4, p$ $<0.001]$ and SI $[F(3,164)=51, p<0.0001]$ upon \%correct.
A. Variable duration

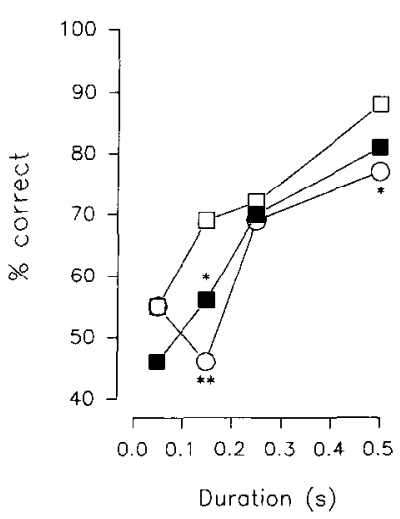

B. Voriable intensity

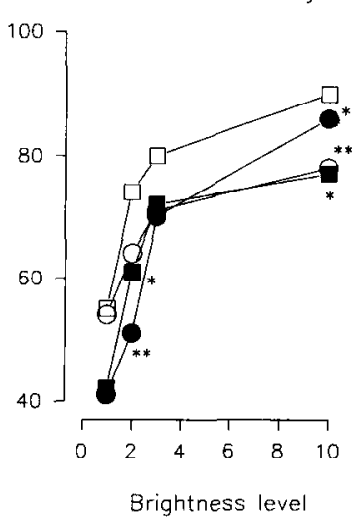

C. White noise distraction

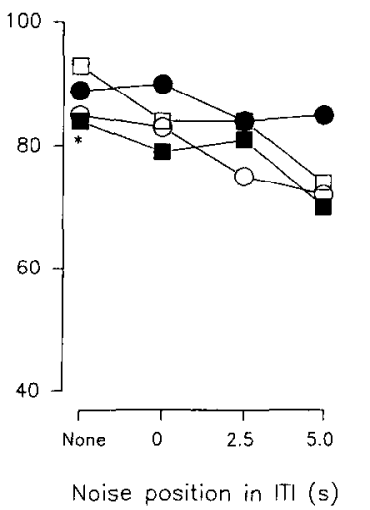

Figure 3. Effect of age on performance in the five-choice task under various parameter changes. $A$, Variable stimulus duration. $B$, Variable stimulus intensity. $C$, White noise distractor. ( $\square$ ) Y group, ( $\square$ ) MA group, $(O) \mathrm{AG}(0.5$ $\mathrm{sec})$, (O) AG(1 sec). See Materials and Methods for further description of parameter changes. ${ }^{*} p<0.05{ }^{* *} p<0.01$ vs $\mathrm{Y}$ group (Tukeys $t$ test). 
Table 1. Effect of variable stimulus duration or intensity upon various performance measures in the serial five-choice task

\begin{tabular}{|c|c|c|c|c|c|c|c|c|}
\hline \multirow[b]{3}{*}{$\%$ Correct } & \multicolumn{4}{|c|}{ Stimulus duration } & \multicolumn{4}{|c|}{ Stimulus intensity } \\
\hline & \multirow{2}{*}{$\begin{array}{l}\text { Young } \\
71 \pm 2\end{array}$} & \multirow{2}{*}{$\frac{\text { Middle-aged }}{64 \pm 2}$} & \multicolumn{2}{|c|}{ Aged $(0.5 \mathrm{sec})$ Aged $(1 \mathrm{sec})$} & \multirow{2}{*}{$\begin{array}{l}\text { Young } \\
75 \pm 2\end{array}$} & \multirow{2}{*}{$\frac{\text { Middle-aged }}{63 \pm 3^{*}}$} & \multicolumn{2}{|c|}{ Aged $(0.5 \mathrm{sec})$ Aged $(1 \mathrm{sec})$} \\
\hline & & & $63 \pm 4$ & $62 \pm 4$ & & & $67 \pm 5^{*}$ & $62 \pm 3 * *$ \\
\hline \%Misses & $12 \pm 3$ & $15 \pm 3$ & $19 \pm 4$ & $28 \pm 4^{* *}$ & $7 \pm 1$ & $15 \pm 2$ & $16 \pm 6$ & $17 \pm 3$ \\
\hline Correct Lat (sec) & $0.55 \pm 0.04$ & $0.60 \pm 0.05$ & $0.70 \pm 0.06$ & $0.70 \pm 0.04$ & $0.64 \pm 0.02$ & $0.63 \pm 0.03$ & $0.64 \pm 0.05$ & $0.82 \pm 0.04^{* *}$ \\
\hline Mag. Lat (sec) & $1.2 \pm 0.05$ & $1.1 \pm 0.05$ & $1.2 \pm 0.07$ & $1.2 \pm 0.06$ & $1.2 \pm 0.07$ & $1.0 \pm 0.04 *$ & $1.2 \pm 0.05$ & $1.2 \pm 0.08$ \\
\hline Prem. responses & $17 \pm 2$ & $18 \pm 2$ & $12 \pm 2$ & $14 \pm 2$ & $27 \pm 5$ & $28 \pm 6$ & $23 \pm 4$ & $23 \pm 4$ \\
\hline
\end{tabular}

* and $* *$ indicate significant differences $(p<0.05$ and 0.01 , respectively) from young rats under the corresponding conditions.

In general, the effects of age were apparent under all levels of stimulus intensity, the $\mathrm{Y}$ group performing with higher accuracy at each level. Collapsing the data across stimulus intensity revealed a main effect of age upon overall \%correct $[F(3,41)=$ 3.8, $p<0.05]$; $\mathrm{AG}(1 \mathrm{sec}), \mathrm{AG}(0.5 \mathrm{sec})$, and MA each being significantly worse than $\mathrm{Y}$ (see Table 1).

There was an effect of age upon correct latency $[F(3,41)=$ $5.6, p<0.01]$, resulting from the increased response time of AG( $1 \mathrm{sec})$ compared with $Y$. There was also an effect of age upon magazine latency $[F(3,41)=3.0, p<0.05]$; however, this was as a result of a reduced latency to retrieve the food reward by MA compared with Y. There was no effect of age upon overall \%omissions, premature responses, perseverative response or panel pushes (see Table 1).

Variable ITI. Two separate experiments were conducted, examining the interaction between age and short variable ITIs (2$5 \mathrm{sec}$ ) and long variable ITIs $(5-8 \mathrm{sec})$. In each experiment significant main effects of age were found [short ITI: $F(3,160)$ 3.8; long ITI: $F(3,160)-3.8$; both $\rho<0.05$ ]. However, in each case, post hoc analysis revealed no age related impairment, rather the $A G(1 \mathrm{sec})$ animals performed significantly better than all other groups at the extreme ITI durations, i.e, 2 and $8 \mathrm{sec}$. Presumably, the longer stimulus duration required for these animals afforded some protection against the variable ITI. In each experiment, both $\mathrm{AG}(0.5 \mathrm{sec})$ and $\mathrm{AG}(1 \mathrm{sec})$ were slower to

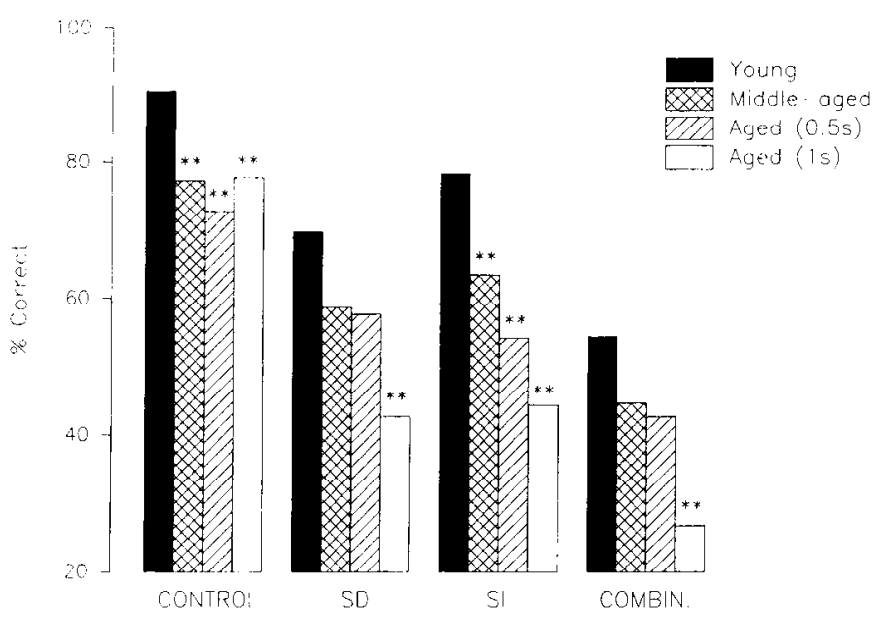

Figure 4. Effect of age on performance in the five-choice task under the test condition of reduced stimulus duration $(S D, 0.15 \mathrm{sec})$, stimulus intensity ( $S I, 45 \mathrm{lux}$ ), and reduced stimulus duration and intensity ( $\mathrm{Com}$ bin). Control reflects the standard condition of $(0.5 \mathrm{sec}$ SD and 575 lux). The $A G(1 \mathrm{sec})$ group were tested under combinations of $0.25 \mathrm{sec}$ and 1 sec SD. ${ }^{*} p<0.05 * * p<0.01$ vs $\mathrm{Y}$ group (Tukeys $t$ test). make a correct reponse (but not to collect the food reward), compared to $\mathrm{Y}$ rats (data not shown).

Interpolation of a white noise distractor. This experiment used 6 rats from $A G(0.5), 11$ rats from $A G(1 \mathrm{sec}), 14$ rats from $\mathrm{MA}$, and 12 rats from $\mathrm{Y}$. The effects of white noisc distraction on accuracy are illustrated in Figure $3 c$. There was a main effect of noise $[F(3,156)=5.3, p<0.01]$ and age $[F(3,156)=6.56$, $p<0.001]$ upon \%correct. Thus, the interpolation of a burst of white noise into the ITI introduced a modest impairment of choice accuracy; however, the magnitude of this impairment was similar across groups. Post hoc analysis revealed a difference at only one comparison point: under control conditions MA were less accurate than $\mathrm{Y}(p<0.05)$. There was no effect of age upon total \%correct.

There was a significant effect of age upon correct latency $[F(3.39)=10.6, p<0.0001]$, as both $\operatorname{AG}(0.5 \mathrm{sec})(0.63 \pm 0.05$ sec, $p<0.05)$ and $\mathrm{AG}(1 \mathrm{sec})(0.72 \pm 0.04 \mathrm{sec}, p<0.01)$, but not MA, were slower than $\mathrm{Y}(0.52 \pm 0.03 \mathrm{sec})$. MA rats were significantly quicker to retrieve the food reward than all other groups. There was also an age-related decrease in the total number of premature responses $[F(3,39)=4.2, p<0.05]$; $\mathrm{AG}(0.5$ sec) $(9 \pm 4, p<0.05)$ and $\mathrm{AG}(1 \mathrm{sec})(10 \pm 5, p<0.01)$ compared with Y $(30 \pm 7)$. Howcver, there were no effects of age upon \%omissions, the total number of perseverative responses or panel pushes.

Combinations of variable stimulus duration with variable stimulus intensity. This experiment used 6 rats from $\mathrm{AG}(0.5)$, 10 rats from $\mathrm{AG}(1 \mathrm{sec}), 14$ rats from $\mathrm{MA}$, and 13 rats from $\mathrm{Y}$. The effects of the combination of standard or reduced SD with standard or reduced SI are illustrated in Figure 4. There were significant main effects of age $[F(3,156)=19.9, p<0.0001]$ and parameter changc $[F(3,156)=53, p<0.0001]$ upon \%correct. Post hoc analysis revealed significant age-related differences from group $Y$ under all test conditions. When the data was collapsed over all trials, there was a highly significant agerelated reduction in total \%correct $[F(3,39)=13.2, p<0.0001]$, from $74 \pm 1 \%(\mathrm{Y})$ to $61 \pm 3 \%(\mathrm{MA}, p<0.01), 57 \pm 4 \%$ $\mathrm{AG}(0.5 \mathrm{sec}), p<0.01)$ or $48 \pm 4 \%$ [AG(1 sec), $p<0.01]$.

There was a significant effect of age upon the total \%omissions $[F(3,39)=7, p<0.001]$, with $\mathrm{AG}(1 \mathrm{sec})(28 \pm$ $5 \%, p<0.01)$ greater than $\mathrm{Y}(8 \pm 3 \%)$. There was also an agerelated effect upon correct latency $[F(3,39)=12.6, p<0.0001]$ as $\mathrm{AG}(0.5 \mathrm{sec})(0.72 \pm 0.05 \mathrm{sec}, p<0.05)$ and $\mathrm{AG}(1 \mathrm{sec})(0.90$ $\pm 0.07 \mathrm{sec}, p<0.01)$ were significantly slower than $\mathrm{Y}(0.53$ $\pm 0.02 \mathrm{sec})$. There was no effect of age on the number of perseverative responses and panel pushes. Furthermore, magazine latency was similar across all groups. 

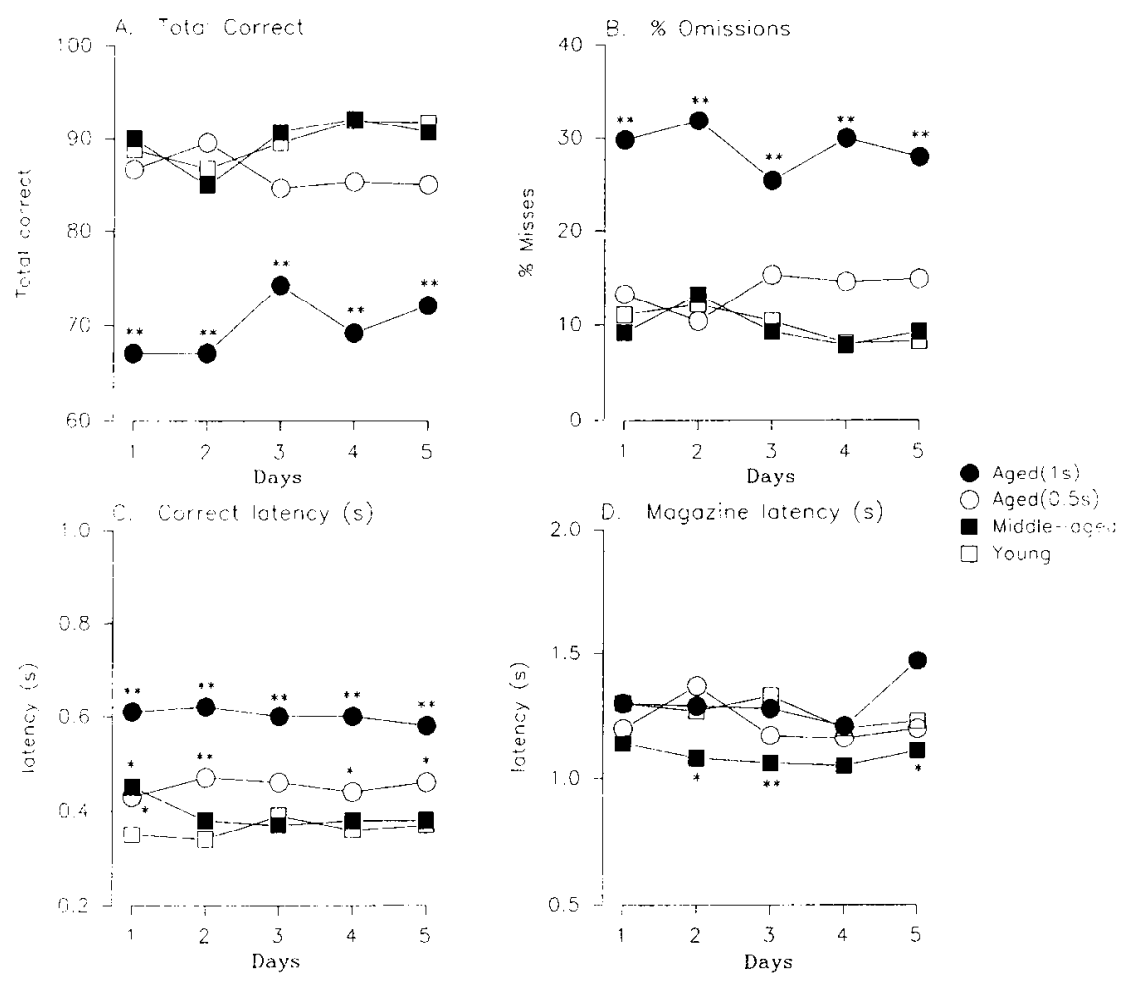

Figure 5. Effect of age on performance over $5 \mathrm{~d}$ continual testing under standard test conditions in the onechoice task. ( $\square$ ) Y group, ( $\square$ ) MA group, (O) $\mathrm{AG}(0.5 \mathrm{sec})$, (O) $\mathrm{AG}(1$ sec). Data shown is for $(A)$ total correct, $(B)$ percentage omissions, $(C)$ correct latency $(\mathrm{sec}),(D)$ magazine latency $(\mathrm{sec}) .{ }^{*} p<0.05{ }^{* *} p<0.01$ vs $\mathrm{Y}$ group (Tukeys $t$ test)

\section{Simple reaction time task}

\section{Performance under standard conditions}

This experiment used 7 rats from $A G(0.5), 10$ rats from $A G(1$ sec), 14 rats from MA, and 13 rats from $Y$. The data for performance in the SRTT over a 5 d period is illustrated in Figure $5 a-d$. There was an effect of age upon total correct $[F(3,198)$ $=33, p<0.0001$, Fig. 5a]. The reduction in total correct for $\mathrm{AG}(1 \mathrm{sec})$ was a result of an increase in \%omissions $[F(3,198)$ $=31, p<0.001$, Fig. 5b]. There were no differences between the other groups of rats. There was an age-dependent increase in correct latency $[F(3,198)=51, p<0.0001$, Fig. $5 c]$ with both $\mathrm{AG}(0.5)$ and $\mathrm{AG}(1 \mathrm{sec})$, but not $\mathrm{MA}$, significantly slower than group Y. As on several occasions in the 5-CSRT task, MA rats were significantly quicker to retrieve the food reward $[F(3,198)=8.8, p<0.0001$, Fig. $5 d]$.

\section{Behavioral challenges}

Reduced stimulus duration. There were significant effects of both age $[F(3,80)=16.3, p<0.0001]$ and $\operatorname{SD}[F(1,80)=7.5$, $p<0.01]$ upon total correct; however, only $\mathrm{AG}(1 \mathrm{sec})$ were significantly worse than $Y$ (see Table 2$)$. AG( 1 sec) $(35 \pm 5, p$ $<0.01)$ rats, but not $\mathrm{MA}$ or $\mathrm{AG}(0.5 \mathrm{sec})$, also missed signifi-

Table 2. Effect of variable stimulus duration or intensity upon total correct in a simple reaction time (SRT) task

\begin{tabular}{|c|c|c|c|c|}
\hline & \multicolumn{2}{|c|}{ Stimulus duration } & \multicolumn{2}{|c|}{ Stimulus intensity } \\
\hline & $0.5 \mathrm{sec}$ & $0.15 \mathrm{sec}$ & 575 lux & $45 \operatorname{lux}$ \\
\hline Young & $46 \pm 2$ & $42 \pm 2$ & $46 \pm 1$ & $44 \pm 1$ \\
\hline Middle-aged & $46 \pm 1$ & $43 \pm 1$ & $48 \pm 1$ & $44 \pm 1$ \\
\hline Aged $(0.5 \mathrm{sec})$ & $44 \pm 2$ & $40 \pm 3$ & $43 \pm 3$ & $40 \pm 3$ \\
\hline Aged $(1 \mathrm{sec})$ & $35 \pm 3^{* *}$ & $30 \pm 3^{* *}$ & $38 \pm 3^{* *}$ & $38 \pm 2 *$ \\
\hline
\end{tabular}

$*$ and $* *$ indicate significant differences $(p<0.05$ and 0.01 , respectively) from young rats under the corresponding conditions. cantly more trials than $\mathrm{Y}(12 \pm 3)$. Both groups of aged rats were significantly slower than $\mathrm{Y}$ to make a response, i.e., 0.45 $\pm 0.02 \mathrm{sec}[\mathrm{AG}(0.5 \mathrm{sec}), p<0.05]$ and $0.55 \pm 0.03 \mathrm{sec}[\mathrm{AG}(1$ $\mathrm{sec}), p<0.01]$ compared with $0.36 \pm 0.03 \mathrm{sec}(\mathrm{Y})$. There were no significant effects on other measures of performance.

Reduced stimulus intensity. There was a main effect of age $[F(3,80)=8.2, p<0.001]$, but not SI, on total correct. AG(1 $\mathrm{sec})$, but not $\mathrm{MA}$ or $\mathrm{AG}(0.5 \mathrm{sec})$, were significantly worse than $\mathrm{Y}$ under both SI conditions $(p<0.05-0.01$, see Table 2$)$. AG( 1 sec) $(24 \pm 5, p<0.01)$, but not MA $(8 \pm 1)$ or $\mathrm{AG}(0.5 \mathrm{sec})$ $(17 \pm 5)$, also missed significantly more trials than $Y(10 \pm 3)$ and were slower to make a response $[F(3,40)=8.2, p<0.001]$ than $Y(0.41 \pm 0.04 \mathrm{sec}, \mathrm{Y}$ compared with $0.62 \pm 0.04 \mathrm{~s}, \mathrm{AG}(1$ $\mathrm{sec}), p<0.01]$. There were no effects on other measures of performance, except that MA were significantly quicker to retrieve the food reward than the other age groups (data not shown).

\section{Effects of cholinergic drugs upon performance in the five-choice serial reaction time task}

Tacrine $(3 \mathrm{mg} / \mathrm{kg}$ )

Thirteen MA rats were used in this experiment, the data from which is illustrated in Figure $6 A$. A significant main effect of tacrine $[F(1,12)=6.6, p=0.02]$ and trial type $[F(3,36)=36.4$, $p<0.01$ ] were found, but no tacrine $\times$ trial type interaction $[F(3,36)=2.0, \mathrm{NS}]$. Post hoc comparisons revealed that tacrine significantly increased choice accuracy under standard condition (CONTROL) and reduced stimulus duration (SD). No other performance measures were affected by tacrine pretreatment, e.g, correct latency: vehicle $0.7 \pm 0.03 \mathrm{~s}$, tacrine $0.7 \pm 0.05 \mathrm{sec}$.

\section{Scopolamine (0.01-0.075 $\mathrm{mg} / \mathrm{kg}$ s.c.)}

This experiment used 8 rats from $\mathrm{Y}$ and 12 rats from MA, and the data is illustrated in Figure $6 B$ and Table 3 . There was a significant main effect of scopolamine $[F(3,76)=4.5, p<0.01]$ 
Figure 6. A, Effect of tacrine on performance of $\mathrm{MA}$ rats in the serial fivechoice task under the condition of reduced stimulus duration ( $S D, 0.15 \mathrm{sec})$, stimulus intensity ( $S I, 45$ lux), and reduced stimulus duration and intensity (Combin). Control reflects the standard condition of $(0.5 \mathrm{sec}$ SD and $575 \mathrm{lux})$. ${ }^{*} p<.05$ vs vehicle control. $B$, Effect of scopolamine pretreatment on the performance of $\mathrm{Y}$ and $\mathrm{MA}$ rats under standard test conditions in the fivechoice task. ${ }^{*} p<0.05{ }^{* *} p<0.01 \mathrm{vs}$ vehicle pretreatment, $+p<0.05++p$ $<0.01$ vs $Y$ group at respective drug pretreatment (Tukeys $t$ test). $C$, Effect of mecamylamine pretreatment on the performance of $\mathrm{Y}$ and MA rats under standard test conditions in the fivechoice task. ${ }^{*} p<0.05{ }^{* *} p<0.01$ vs vehicle pretreatment (Tukeys $t$ test).
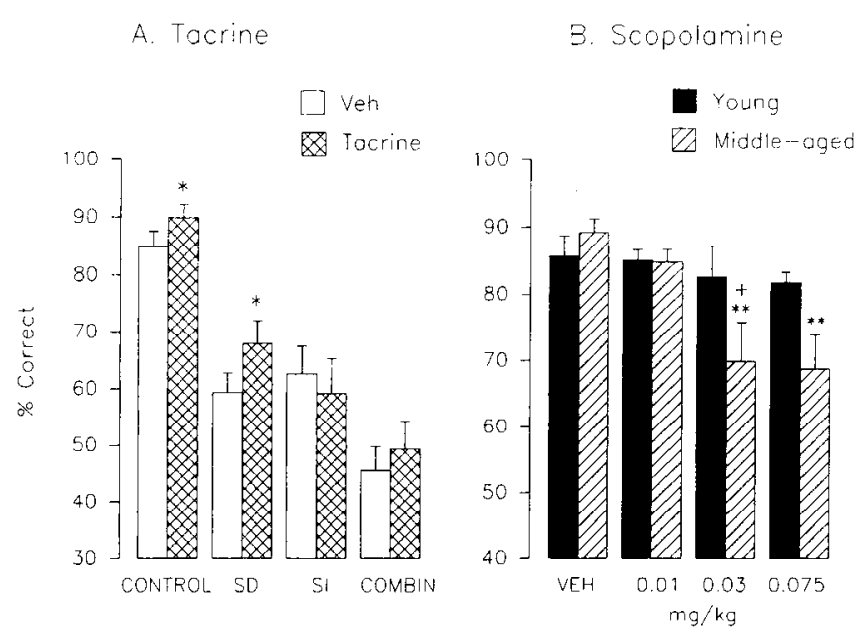

upon \%correct, and a near significant effect of age $[F(1,76)=$ $3.1, p=0.08]$. This was a result of a reduction in \%correct for group MA following 0.03 and $0.075 \mathrm{mg} / \mathrm{kg}$ scopolamine compared with VEH $(p<0.01)$ and compared with $\mathrm{Y}(p<0.05$, for $0.03 \mathrm{mg} / \mathrm{kg}$ scopolamine only, Fig. $6 B$ ). There were similar age-related effects of scopolamine upon \%omissions, correct, and magazine latency (Table 3), but not on any other measures (data not shown).

\section{Mecamylamine $(0.3-5 \mathrm{mg} / \mathrm{kg}$ s.c.)}

There was a main effect of mecamylamine $[F(4,90)=4.4, p<$ $0.01]$, but not age $[F(1,90)=0.7, p>0.05]$ upon \%correct (Fig. 6C). There was an age-related effect of mecamylamine upon \%omissions, such that omissions were significantly higher following mecamylamine in MA than Y (Table 4). Correct latency (but not magazine latency) was increased by mecamylamine treatment in both $\mathrm{Y}$ and MA groups, although this effect was more pronounced in the latter. There were no other agerelated differences in the effects of mecamylamine.

\section{Locomotor activity response to a novel environment}

The number of rats in this study were: Y (8), MA (8), AG(0.5 sec) (7), AG(1 sec) (8). This allowed us to counterbalance the testing of Y, MA, AG groups. The data is shown in Figure $7 A$. There were main effects of both age $[F(3,324)=59, p<0.001]$ and time bin $[F(11,324)=42, p<0.001]$ upon total counts, but no interaction $[F(33,324)=0.96, p>0.05]$. Post hoc analysis revealed significantly lower activity for MA at the 15, 25, 50 , and 60 min time bins compared with $\mathrm{Y}(p<0.05-0.01)$. Both groups of aged rats had a lower level of activity over the majority of the time bins compared with $\mathrm{Y}(p<0.05-0.01)$. Analysis of the accumulated activity for the whole period revealed a significant effect of age $[F(3,27)=13.3, p<0.0001]$, with an age-related reduction in LMA (Fig. $7 B$ ).

\section{Discussion}

Effects of age upon acquisition of the 5-CSRT task

A major finding of this study was that $\mathrm{Y}, \mathrm{MA}$, and at least a subgroup of aged rats were successfully trained in the 5-CSRT task. However, there was a clear effect of age upon the acquisition: MA and aged rats required more trials to reach criterion at each training stage. There are two distinct stages of training; the initial acquisition of the stimulus light-reward association, and a second stage wlien the SD is reduced to $1 \mathrm{sec}$ or below, which specifically places a greater attentional demand on the animals. Together, each revealed a very clear age-related increase in the number of training sessions to criterion.

The deficit in the stimulus light-reward association may be the result of a number of factors. At the beginning of training, MA and aged rats were significantly heavier than $Y$, and all rats had been food restricted for only 1 week prior to training. It is possible that MA and aged rats were less motivated to learn the task as they lost comparatively less body wcight than $Y$ rats. However, even at this stage, there were no apparent differences in the latency to retrieve the food reward, and the rewards were always retrieved, indicating a similar level of motivation for each group. A second, more likely contribution to the acquisition deficit relates to the reduced exploratory drive of MA and aged, compared to $\mathrm{Y}$ rats, rendering these animals less likely to make a correct response by chance. This was seen in both the operant

Table 3. The effects of scopolamine upon \%omissions, correct and magazine latencies (sec) for groups $\mathrm{Y}$ (young) and MA (middle-aged) under standard conditions

\begin{tabular}{|c|c|c|c|c|c|c|}
\hline \multirow[b]{2}{*}{$\mathrm{mg} / \mathrm{kg}$} & \multicolumn{2}{|c|}{ \%Omissions } & \multicolumn{2}{|c|}{ Correct latency (sec) } & \multicolumn{2}{|c|}{ Magazine latency (sec) } \\
\hline & $\mathrm{Y}$ & MA & $\mathrm{Y}$ & MA & $\mathrm{Y}$ & MA \\
\hline VEH & $8 \pm 3$ & $4 \pm 1$ & $0.60 \pm 0.06$ & $0.53 \pm 0.03$ & $1.29 \pm 0.10$ & $1.22 \pm 0.07$ \\
\hline 0.01 & $4+1$ & $12 \pm 6$ & $0.54 \pm 0.02$ & $0.61 \pm 0.04$ & $1.32 \pm 0.10$ & $1.31 \pm 0.08$ \\
\hline 0.03 & $7 \pm 3$ & $30 \pm 8 \ddagger^{* *}$ & $0.59 \pm 0.07$ & $0.74 \pm 0.07 \dagger * *$ & $1.46 \pm 0.13$ & $2.23 \pm 0.72 * *$ \\
\hline 0.075 & $16 \pm 3$ & $44 \pm 7 \neq * *$ & $0.58 \pm 0.03$ & $0.81 \pm 0.11+* *$ & $1.46 \pm 0.11$ & $1.71 \pm 0.11 *$ \\
\hline
\end{tabular}

* and $* *$ indicate significant differences $(p<0.05$ and 0.01 , respectively) from VEH, while $\dagger$ and $\ddagger$ indicate significant differences ( $p<0.05$ and 0.01 , respectively) from the corresponding treatment for group Y. 
Table 4. The effects of mecamylamine upon \%omissions, correct and magazine latencies (sec) for groups $Y$ (young) and MA (middle-aged) under standard conditions

\begin{tabular}{|c|c|c|c|c|c|c|}
\hline \multirow[b]{2}{*}{$\mathrm{mg} / \mathrm{kg}$} & \multicolumn{2}{|c|}{$\%$ Omissions } & \multicolumn{2}{|c|}{ Correct latency (sec) } & \multicolumn{2}{|c|}{ Magazine latency (sec) } \\
\hline & $\bar{Y}$ & MA & $\bar{Y}$ & MA & $\mathrm{Y}$ & MA \\
\hline VEH & $6 \pm 2$ & $5 \pm 1$ & $0.55 \pm 0.06$ & $0.55 \pm 0.04$ & $1.31 \pm 0.09$ & $1.37 \pm 0.09$ \\
\hline 0.3 & $5 \pm 2$ & $8 \pm 2$ & $0.56 \pm 0.05$ & $0.59 \pm 0.05$ & $1.35 \pm 0.11$ & $1.31 \pm 0.08$ \\
\hline 1.0 & $5 \pm 2$ & $12 \pm 3$ & $0.57 \pm 0.06$ & $0.65 \pm 0.05$ & $1.44 \pm 0.14$ & $1.36 \pm 0.09$ \\
\hline 3.0 & $17 \pm 4^{*}$ & $27 \pm 4 \uparrow * *$ & $0.83 \pm 0.05^{* *}$ & $0.82 \pm 0.06 * *$ & $1.45 \pm 0.12$ & $1.42 \pm 0.08$ \\
\hline 5.0 & $40 \perp 3 * *$ & $53 \perp 3 \neq * *$ & $0.67 \perp 0.04$ & $0.93 \pm 0.16^{* *}$ & $1.40 \pm 0.12$ & $1.68 \pm 0.17$ \\
\hline
\end{tabular}

$*$ and $* *$ indicate significant differences $(p<0.05$ and 0.01 , respectively) from $\mathrm{VEH}$, while $\dagger$ and $\ddagger$ indicate significant differences $(p<0.05$ and 0.01 , respectively) from the corresponding treatment for group Y.

chamber (visual inspection, not quantified) and within a novel open field arena.

The slower acquisition of the stimulus light-reward association in the MA and $A G$ rats may be related to the unpredictable location of the stimulus presentation. There are many studies showing an age-related impairment of acquisition of either spatial (Rapp et al., 1987; Gage et al., 1988; Fischer ct al., 1991; Pitsikas and Algeri, 1992; Van der Staay and de Jonge, 1993) or operant (Dunnett et al., 1988) tasks, but in each case the associative stimulus is presented in a more predictable manner than in the five-choice task. Thus, the need to adapt to a variety of stimulus locations is likely to require attentional as well as mnemonic processes. Deficits in either of these functions in the MA and AG rats are also likely to contribute to the slower acquisition rates during the early phase of training. Training different age groups of naive rats in the more predictable SCRT time task may provide valuable information about this.

The second part of the training procedure, which involves the gradual reduction of $S D$ to 1 and $0.5 \mathrm{sec}$, represents the first time where a response is generally made in the absence of the light stimulus (i.e., correct latency of greater than $0.5 \mathrm{sec}$ ). It is perhaps important that the clearest age-related effects were seen at this stagc. MA and both groups of aged rats required significantly more sessions to reach criterion than $Y$. This may represent an age-related effect upon attention. However, reducing SD may introduce a short-term memory component to the task, for the rat must detect the light stimulus and remember which of the five holes to respond to (Muir et al., 1994) and make this task analogous to a delayed response task (Dunnett et al., 1988). Presumably, as the response latencies for all groups are reduced, the memory component to this task will become negligible. Nonetheless, the greater response latencies for aged rats through- out these studies may introduce a small working memory componant to this task compared with both MA and Y.

\section{Effects of age upon baseline performance of the 5-CSRT and SRT tasks}

Once trained, there were effects of aging upon baseline performance of the 5-CSRT task. The effects on \%omissions and correct latency were the most robust. Both $\mathrm{MA}$ and $\mathrm{AG}(0.5 \mathrm{sec})$ showed a trend towards a reduced accuracy compared with $\mathrm{Y}$, which reached significance for MA on day 5, although this effect was not apparent in any later sessions run under standard conditions throughout the period of this report (e.g., note vehicle pretreated $\mathrm{Y}$ and MA groups in Fig. $6 B$ and $C$ ). The age-related increase in omissions is unlikely to reflect a reduced motivation, as there were no effects of aging upon reward retrieval latency. In fact, there was a consistent trend for MA to show the fastest magazine latency - a finding for which we have no obvious explanation. Both groups of aged rats were significantly slower to make a correct response than $\mathrm{Y}$, although a similar level of accuracy was achieved, suggesting adoption of a speed/error trade off strategy (Robbins et al., 1989).

Following the behavioral challenges in the 5-CSRT task, rats were trained in the SRT task. There were no differences in the number of correct responses or \%omissions between Y, MA, and $\mathrm{AG}(0.5 \mathrm{sec})$. However, $\mathrm{AG}(1 \mathrm{sec})$ rats made a large number of errors of omission (approximately 30\%). Compared with the 5-CSRT task, the increase in \%omissions for AG(1 sec) and the similar levels for $\mathrm{AG}(0.5 \mathrm{sec}), \mathrm{MA}$, and $\mathrm{Y}$ rats was surprising. It was expected that omissions would fall as the numbers of response choices was reduced from five to one. The greatly reduced LH (from 5 to $2 \mathrm{sec}$ ) and/or the variable ITI (from 5 to 4.25-5.75 sec) may explain these findings. However, these
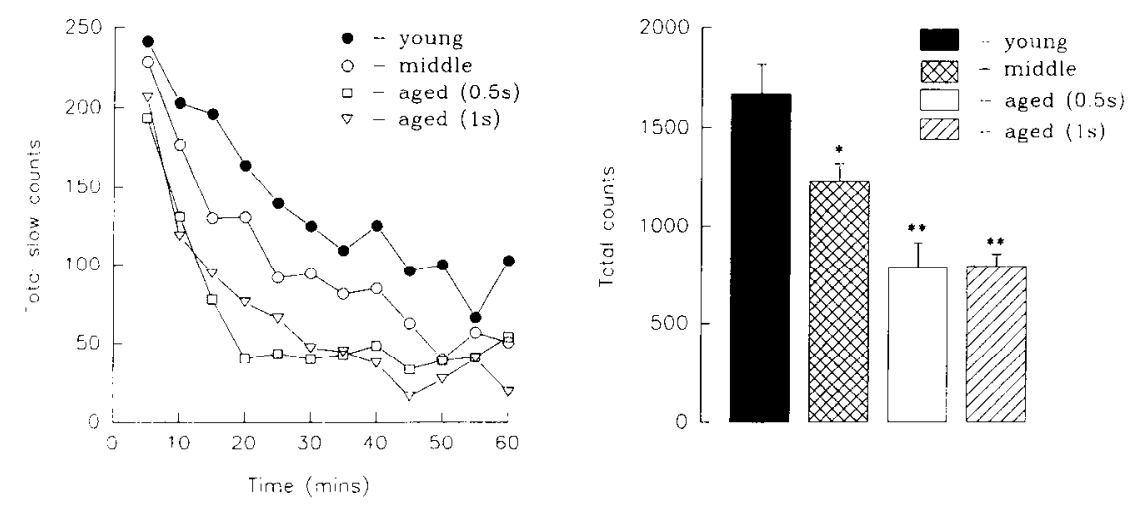

Figure 7. Effect of age on general activity within an open field chamber. $A$, Temporal distribution of activity counts over the $60 \mathrm{~min}$ test period. (O) Y, (O) MA, ( $\square)$ AG $(0.5 \mathrm{sec}),(\nabla)$ AG $(1 \mathrm{sec})$. $B$, Total number of activity counts recorded during the 60 min test period. ${ }^{*} p<0.05{ }^{*} p<0.01$ vs Y group (Tukeys $t$ test). 


\begin{tabular}{|c|c|c|c|}
\hline & MA & $\mathrm{AG}(0.5)$ & $A G(1)$ \\
\hline \multicolumn{4}{|l|}{ A. Choice accuracy } \\
\hline Five-choice task: Standard Conditions (SI: 575 lux, SD: $0.05 \mathrm{sec}$ ) & NSE & $\mathrm{NSE}^{a}$ & NSE \\
\hline Variable stimulus duration (SD: $0.05-0.5 \mathrm{sec}$ ) & I & I & - \\
\hline Variable stimulus intensity (SI: 16-575 lux) & I & 1 & I \\
\hline Combination reduced SD/SI & I & I & I \\
\hline Noise distractor & NSE & NSE & NSE \\
\hline Simple reaction time task & NSE & NSE & I \\
\hline \multicolumn{4}{|l|}{ B. Performance measures } \\
\hline Omissions & NSE & NSE & increased \\
\hline Correct latency & NSE & increased & increased \\
\hline Magazine latency & $\mathrm{NSE}^{b}$ & NSE & NSE \\
\hline
\end{tabular}

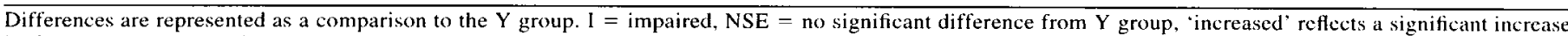
in the measure compared to $\mathrm{Y}$ rats. Panel pushes, perseverative and premature responses were either not affected or inconsistently affected by age.

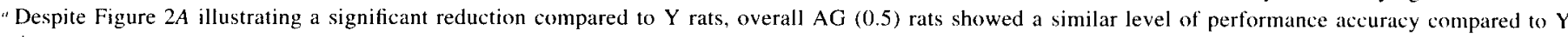
rats.

"MA rats tended to be faster than $\mathrm{Y}$ group.

changes were necessary to introduce a mild degree of stimulus unpredictability, otherwise the animals could simply respond without having to attend to the stimulus.

Similar to the 5-CSRT task, in the SRT task there was an agerelated increase in correct latencies; both groups of aged rats being slower than $Y$; although again, there were no age-related differences in magazine latency. It is also important to note that compared with the SRT task, all rats were slower to make a correct response in the 5-CSRT task. This suggests that correct latency is sensitive to increased attentional load caused by increased response choices, i.e., greater demands upon signal detection/processing and decision processing.

\section{The effects of challenge with variable stimulus parameters}

Table 5 summarizes the effect of age on responding to the various stimulus conditions. For $\mathrm{Y}, \mathrm{MA}$, and $\mathrm{AG}(0.5 \mathrm{sec})$ rats at least, reducing SD from $0.5 \mathrm{sec}$ to $0.15 \mathrm{sec}$, or SI from $575 \mathrm{lux}$ to $45 \mathrm{lux}$, exacerbated age-related differences in response accuracy in the 5-CSRT task, but not in the SRT task. Robbins and co-workers have suggested that increased sensitivity to reduced SI is indicative of effects upon visual function, rather than attentional processes (Robbins et al., 1989; Muir et al., 1992a,b). However, we found a clear dissociation in relative sensitivity of the different age groups to variations in SI or SD between the SRT and 5-CSRT tasks. For example, the AG(0.5 sec) group were significantly impaired relative to $\mathrm{Y}$, when $\mathrm{SD}$ was reduced from 0.5 to $0.15 \mathrm{sec}$ in the 5-CSRT, but not in the SRT task. These findings suggest that the age-related increase in sensitivity to variahle SD and SI are a result of impaired attentional processes rather than sensorimotor deficits. For AG $(1 \mathrm{sec})$, however, the effects in the 5-CSRT task were mirrored to some degree in the SRT task, suggesting factors other than attention, possibly sensory function, are involved.

Increased sensitivity to reduced SD (but not SI), and also the adoption of a speed/error tradeoff stategy has been reported following disruption of the forebrain cholinergic system (Robbins et al., 1989; Muir et al., 1994), a system that is reported to be degenerated in aged rats (Flood and Coleman, 1988; Fischer et al., 1992; Smith et al., 1993). Disrupting the cholinergic system exacerbates distraction caused by a burst of loud white noise
(Robbins et al., 1989; Muir et al., 1992a; Kirkby et al., 1994; Jones and Higgins, 1995). However, there was no evidence of increased distractibility with aging in the present study. The \%correct for $\mathrm{Y}, \mathrm{MA}$, and $\mathrm{AG}(0.5 \mathrm{sec})$ rats each showed a similar rate of decline and was particularly apparent when the white noise was simultaneous with the light stimulus. Taken together, these findings illustrate some dissociations between the pattern of changes produced by cholinergic lesions and aging. Presumably this reflects the involvement of variety of neurotransmitter systems, including the cholinergic system, in age-related deficits (Arnsten and Goldman-Rakic, 1985; Flood and Coleman, 1988; Gallagher et al., 1990; Fischer et al., 1992; Gallagher and Nicolle, 1993; Nagahara et al., 1993; Smith et al., 1993), and/or that age-associated changes in cholinergic function are not likely to be as severe as that produced by selective pharmacological or surgical disruption of this system. In general, it appears that in rats, age-associated reductions in ChAT activity are not as extensive as those induced by basal forebrain neurotoxin lesions (e.g., see Dunnett et al., 1991; Fischer et al., 1991; Higgins et al, 1995).

\section{The influence of cholinergic drugs upon performance}

The ability of the anticholinesterase drug tacrine to cause a small improvement in the performance of MA was of interest for two reasons. First, it implies that the performance decline of MA rats is at least partially attributable to a cholinergic deficit. It was interesting to note that in the combination procedure used, tacrine only improved performance under conditions of reduced stimulus duration, not intensity. This is consistent with the relative sensitivities of these manipulations to cholinergic lesions (Robbins et al,. 1989; Muir et al., 1994). Second, the present findings are comparable with those of Sahakian and co-workers (1993) who reported improved performance in the 5-CSRT task for some AD patients following tacrine. In previous studies, in a very wide range of conditions, tacrine has consistently failed to influence the performance accuracy of young rats (Jones et al., unpublished data). The effects of tacrine in MA rats suggests that this test may have some predictive value.

Low doses of scopolamine selectively impaired the accuracy of MA but not $\mathrm{Y}$ rats under standard conditions. This finding is 
particularly notable because of the consistent lack of effects upon accuracy of younger rats to scopolamine under these conditions (Jakala et al., 1992; Jones and Higgins, 1995). The reductions in choice accuracy may simply reflect a more generalized performance disruption as evidenced by the increased omissions and latency measures in MA rats. However, against this, mecamylamine caused similar changes in omissions in $\mathrm{Y}$ and MA rats, although accuracy was reduced by only $5-10 \%$. Similar age associated changes in the magnitude of a scopolamine-induced impairment in spatial learning has recently been reported (Nilsson and Gage, 1993). Therefore, it is quite likely that the increased sensitivity to cholinergic blockade is a reflection of an age associated decline in the functioning of forebrain cholinergic systems (Flood and Coleman, 1988; Fischer et al., 1991; Smith et al., 1993); however, further study is required.

Mecamylamine also impaired performance accuracy, although the effect was similar in both $\mathrm{Y}$ and MA rats, and of smaller magnitude compared to scopolamine. These results indicate a role for both nicotinic as well as muscarinic cholinoceptors in attentional processing. Mecamylamine impairs performance of rodents in a variety of memory tasks, although studies using tests designed to specitically measure attention have not been conducted to date (Levin, 1992). Our present studies therefore significantly extend this literature. Despite magazine latency being largely unaffected by mecamylamine, the animals were markedly slower to make a correct response, suggesting the adoption of a speed/error tradeoff strategy. In humans, mecamylamine has similarly been reported to slow cognitive performance (Stolerman et al., 1973). An important role of nicotinic cholinergic systems in attention is supported further by clinical studies; the most consistent finding following acute nicotine injection or cigarette smoking effects on cognition is improved vigilance and information processing (Wesnes and Warburton, 1984; Sahakian et al., 1989; Levin, 1992).

\section{Conclusions}

The main finding from the present study is of an age-related impairment in the acquisition and performance in the 5-CSRT task, which was most clearly seen when the duration or intensity of the light stimulus was reduced. For $M \Lambda$ and $\Lambda \mathrm{G}(0.5)$ rats at least, these effects were not mirrored by performance deficits in the SRT task. Together, these observations might indicate an age-related deficit in attention in the rat that can be detected by the five-choice serial reaction time task. Furthermore, the agerelated differences in sensitivity to the effects of tacrine and scopolamine suggests the involvment of the muscarinic cholinergic system in at least some of these deficits.

\section{References}

Albert MS (1988) Special behavioural paradigms in animals to study age-related changes in memory. Neurobiol Aging 9:507-509.

Arnsten AFT, Goldman-Rakic PS (1985) Catecholamines and cognitive decline in aged nonhuman primates. Ann NY Acad Sci 218-234.

Barnes CA (1990) Animal models of age-related cognitive decline. In: Handbook of neuropsychopsychology, Vol 4 (Boller F, Grafman J, eds), pp 169-196. Amsterdam: Elsevier.

Bartus RT, Dean RL, Beer B (1980) Memory deficits in aged cebus monkeys and facilitation with central cholinomimetics. Neurobiol Aging 1:145-152.

Broadbent DE (1971) Decision and stress. New York: Academic.

Capatani E, Della Sala S, Lucchelli F, Soave P, Spinnler H (1988) Perceptual attention in ageing and dementia measured by Gottschaldt's hidden figure test. J Gerontol 6:157-163.

Carli M, Robbins TW, Evenden JL, Everitt BJ (1983) Effects of lesions to ascending noradrenergic neurons on performance on a 5 -choice serial reaction time task in rats: implications for theories of dorsal noradrenergic bundle function based on selective attention and arousal. Behav Brain Res 9:361-380.

Craik FIM, Byrd M (1982) Aging and cognitive deficits. The role of attentional resources. In: Aging and cognitive processes (Craik FIM, Trehub S, eds), pp 191-211. New York: Plenum.

Craik FIM, Simon E (1980) Age differences in memory. The roles of attention and depth processing. In: New directions in memory and aging (Poon LW, Fozard JL, Cermak LS, Arenberg D, Thompson LW, eds), pp 95-111. Hillsdale, NJ: Erlbaum.

Cole BJ, Robbins TW (1989) Effects of 6-hydroxydopamine lesions of the nucleus accumbens septi on performance of a 5-choice serial reaction time task in rats: implications for theories of selective attention and arousal. Behav Brain Res 33:165-179.

Coyle JT, Price DL, Delong MR (1983) Alzheimer's disease: a disorder of cortical cholinergic innervation. Science 217:1184-1190.

Dunnett SB, Evenden JL, Iversen SD (1988) Delay-dependant shortterm memory deficits in aged rats. Psychopharmacology (Berl) 96: $174-180$.

Dunnett SB, Everitt BJ, Robbins TW (1991) The basal forebrain-cortical cholinergic system: interpreting the functional consequences of excitotoxic lesions. Trends Neurosci 14:494-501.

Fischer W, Chen KS, Gage FH, Bjorkland A (1991) Progressive decline in spatial learning and integrity of forebrain cholinergic neurons in rats during aging. Neurobiol Aging 13:9-23.

Flood DG, Coleman PD (1988) Neuron numbers and sizes in aging brain: comparisons of human, monkey, and rodent data. Neurobiol Aging 9:453-463.

Freed DM, Corkin S, Growden JH, Nissen M (1989) Selective attention in Alzheimer's disease: characterizing cognitive subgroups of patients. Neuropsychologia 27:325-339.

Gage FH, Dunnett SB, Bjorklund A (1988) Spatial learning and motor deficits in aged rats. Neurobiol Aging 9:453-463.

Gallagher M, Pelleymounter MA (1988) Spatial learning deficits in old rats: a model for memory decline in the aged. Neurobiol Aging 5:4348.

Gallagher M, Burwell RD, Kodsi MH, McKinney M, Southerland S, Vella-Roundtree L, Lewis MH (1990) Markers for biogenic amines in the aged rat brain: relationship to decline in spatial learning ability. Neurobiol Aging 11:507-514.

Gallagher M, Nicolle MM (1993) Animal models of normal aging: relationship between cognitive decline and markers in hippocampal circuitry. Behav Brain Res 57:155-162.

Gordon B, Carson K (1990) The basis for choice reaction time slowing in Alzheimer's disease. Brain Cogn 13:148-166.

Gower AJ, Lamberty Y (1993) The aged mouse as a model of cognitive decline with special emphasis on studies in NMRI mice. Behav Brain Res 57:163-173.

Grady CL, Grimes AM, Patronas N, Sunderland T, Foster NL, Rapoport SI (1989) Divided attention, as measured by dichotic speech performance, in dementia of the Alzheimer type. Arch Neurol 46:317-320.

Higgins GA, Clapham J, Murray J, Kirkby DL, Rupniak HTR, McCleave H, Barnes JC (1995) Behavioural, neurochemical and morphological changes in aged rats: comparison with basal forebrain and entorhinal cortex lesions. Soc Neurosci Abstr 1995.

Ingram D (1985) Analysis of age-related impairments in learning and memory in rodent models. Ann NY Acad Sci 44:312-331.

Jakala P, Sirvio J, Jolkkonen J, Riekkinen P, Acsady L, Riekkenen P (1992) The effects of p-chlorophenylalanine-induced serotonin synthesis inhibition and muscarinic blockade on the performance of rats in a 5-choice serial reaction time task. Behav Brain Res 51:29-40.

Jones DNC, Higgins GA (1995) Effect of scopolamine on visual attention in rats. Psychopharmacology (Berl) 120:142-149.

Jones DNC, Barnes JC, Kirkby DL, Higgins GA (1993) Behavioural and pharmacological performance disruption in a 5-choice serial reaction time task in rats. J Psychopharmacol (Abstr book) A82.

Kirkby DL, Jones DNC, Higgins GA (1994) Hemicholinium and cognition. J Psychopharmacol (Abstr book) A13.

Levin ED (1992) Nicotinic systems and cognitive function. Psychopharmacology (Berl) 108:417-431.

McGauty J, Sarter M (1995) Behavioral vigilance in rats: task validation and effects of age, amphetamine, and benzodiazepine receptor ligands. Psychopharmacology (Berl) 117:340-357.

McGeer PL, McGeer EG, Suzuki J, Dolman CE, Nagai T (1984) Ag- 
ing, Alzheimer's disease, and the cholincrgic systcm of the basal forebrain. Neurology 34:741-745.

Moore H, Dudchenko P, Bruno JP, Sarter M (1992) Toward modelling age-related changes of attentional abilities in rats: simple and choice reaction time tasks and vigilance. Neurobiol Aging 13:759-772.

Muir JL, Dunnett SB, Robbins TW, Everitt, BJ (1992a) Attentional functions of the forebrain cholinergic system: effects of intraventricular hemicholinium, physostigmine, basal forebrain lesions and intracortical grafts on a multiple-choice serial reaction time task. Exp Brain Res 89:611-622.

Muir JL, Robbins TW, Everitt BJ (1992b) Disruptive effects of muscimol infused into the basal forebrain on conditional discrimination and visual attention: differential interactions with cholinergic mechanisms. Psychopharmacology (Berl) 107:541-550.

Muir JL, Everitt BJ, Robbins TW (1994) AMPA-induced excitotoxic lesions of the basal forebrain: a significant role for the cortical cholinergic stystem in attentional function. J Neurosci 14:2313-2326.

Nagahara AH, Nicolle MM, Gallagher M (1993) Alterations in [ ${ }^{3} \mathrm{H}$ kainate receptor binding in the hippocampal formation of aged LongEvans rats. Hippocampus 3:269-277.

Nilsson OG, Gage FH (1993) Anticholinergic sensitivity in the aging rat septohippocampal system as assessed in a spatial memory task. Neurobiol Aging 14:487-497.

Parasuraman R, Haxby JV (1993) Attention and brain function in $\mathrm{Al}$ zheimer's disease: a review. Neuropsychology 7:242-272.

Parasuraman R, Martin A (1994) Cognition in Alzheimer's disease disorders of attention and semantic knowledge. Curr Opin Neurobiol $4: 237-244$

Parasuraman R, Greenwood PM, Haxby JV, Grady CL (1992) Visuospatial attention in dementia of the Alzheimer type. Brain 115:711733

Perry EK, Tomlinson BE, Blessed G, Bergman K, Gibson PH, Perry RH (1978) Correlation of cholinergic abnormalities with senile plaques and mental test scores in senile dementia. Br Med J 2:14571459.

Pitsikas N, Algeri S (1992) Deterioration of spatial and nonspatial reference and working memory in aged rats: protective effects of lifelong calorie restriction. Neurobiol Aging 13:369-373.

Rapp PR, Rosenberg RA, Gallagher M (1987) An evaluation of spatial information processing in aged rats. Behav Neurosci 101:3-12.

Robbins TW, Everitt BJ, Marston HM, Wilkinson J, Jones GH, Page
KJ (1989) Comparative effects of ibotenic acid- and quisqualic acidinduced lesions of the substantia innominata on attentional function in the rat: further implications for the role of the cholinergic neurons of the nucleus basalis in cognitive processes. Behav Brain Res 35: 221-240.

Sarter M (1994) Neuronal mechanisms of attentional dysfunctions in senile dementia and schizophrenia: two sides of the same coin? Psychopharmacology (Berl) 1 14:539-550.

Sahakian B, Jones G, Levy R, Gray J, Warburton D (1989) The effects of nicotine on attention, information processing and short term memory in patients with dementia of the Alzheimer type. Br J Psychiatry 1 54:797-800

Sahakian BJ, Owen AM, Morant NJ, Eagger SA, Boddington S, Crayton L, Crockcroft HA, Crooks M, Hill K, Levy R (1993) Further analysis of the cognitive effects of tetrahydroaminoacridine (THA) in Alzheimer's disease: assessment of attentional and mnemonic function using CANTAB. Psychopharmacology (Berl) 1 10:395-401

Schneider W, Pimm-Smith M, Worden M (1994) Neurobiology of attention and automaticity. Curr Opin Neurobiol 4:177-182.

Smith ML, Deadwyler SA, Booze RM (1993) 3-D reconstruction of the cholinergic basal forebrain system in young and aged rats. Neurobiol Aging 14:389-392.

Stolerman IP, Goldfarb T, Fink R, Jarvik ME (1973) Influencing cigarette smoking with nicotine antagonists. Psychopharmacology (Berl) 28:247-259.

van der Stay FI, de Jonge $M$ (1993) Fffects of age on water escape behavior and on repeated acquisition in rats. Behav Neurobiol 60: $33-41$.

Vogels OJM, Broere CAJ, Ter Laak HJ, Ten Donkelaar HJ, Nieuwenhuys R, Schulte BPM (1990) Cell loss and shrinkage in the nucleus basalis Meynert complex in Alzheimer's disease. Neurobiol Aging $11: 3-13$.

Voytko M, Olton DS, Richardson RT, Gorman LK, Tobin JR, Price DL (1994) Basal forebrain lesions in monkeys disrupt attention but not learning and memory. J Neurosci 14:167-186.

Wesnes K, Warburton DM (1984) Effects of cigarettes of varying yield on rapid information processing. Psychopharmacology (Berl) 82: 338-342.

Whitehouse PJ, Price DL, Struble RG, Clark AW, Coyle JT, Delong MR (1982) Altheimer's disease and senile dementia: loss of neurons in the basal forebrain. Science 215:1237-1239. 